\title{
Personality and Mental Health
}

Personality and Mental Health

12: 321-333 (2018-11)

Published online 28 August 2018 in Wiley Online Library

(wileyonlinelibrary.com) DOI 10.1002/pmh.1430

\section{Do treatment outcomes differ after 3 months DBT inpatient treatment based on borderline personality disorder subtypes?}

\author{
ELLEN SLEUWAEGEN ${ }^{1,2}$ D, LAURENCE CLAES ${ }^{2,3}$, KOEN LUYCKX $^{3}$, TOM WILDERJANS $^{3,4}$, \\ ANN BERENS ${ }^{1}$ AND BERNARD SABBE ${ }^{1,2}$, ${ }^{1}$ University Department of Psychiatry, Campus \\ Psychiatric Hospital Duffel, Duffel, Belgium; ${ }^{2}$ Faculty of Medicine and Health Sciences, University \\ of Antwerp, Antwerp, Belgium; ${ }^{3}$ Faculty of Psychology and Educational Sciences, KU Leuven, \\ Leuven, Belgium; ${ }^{4}$ Research Group of Methodology and Statistics, Institute of Psychology, Leiden \\ University, Leiden, The Netherlands
}

\begin{abstract}
Heterogeneity in borderline personality disorder (BPD) drives the search for BPD subtypes to optimize the assessment and treatment of these patients. Therefore, the aims of the present study were (1) to replicate previously identified BPD subtypes based on reactive and regulative temperament; (2) to compare them on symptomatology and coping; and (3) to investigate whether these subtypes show different treatment responses after 3 months of inpatient dialectical behaviour therapy (DBT). A total of 145 BPD inpatients were assessed by means of measures of temperament, symptomatology and coping. Through model-based clustering on the Behavioural Inhibition and Behavioural Activation Scales (BISBAS) and Effortful Control Scale (ECS), we identified three BPD subtypes: an Emotional/Disinhibited subtype (15\%, high BAS and low ECS); a Low Anxiety subtype (41\%, low BIS) and an Inhibited subtype (44\%, low BAS). After 3 months of DBT, 75 patients completed the measures for a second time. Repeated measure ANOVAs demonstrated a general improvement on all symptoms and coping strategies. In addition, the BPD subtypes showed trajectory differences in clinical and borderline specific symptomatology and dissociation. These findings indicate that BPD subtypes based on temperament demonstrate different treatment responses, which can contribute to the search of more BPD subtype tailored treatment interventions. (C) 2018 John Wiley $\mathcal{G}$ Sons, Ltd.
\end{abstract}

\section{HIGHLIGHTS}

- Three BPD subtypes were identified based on reactive and regulative temperament: the Emotional/Disinhibited, Inhibited and Low Anxiety subtype.

- All BPD patients improved in symptomatology and coping after 3 months of DBT.

- The three BPD subtypes differed in symptom trajectory after 3 months of DBT.

- In contrast to the other two subtypes, the Low Anxiety subtype did not show a significant decrease in symptomatology. 


\section{Introduction}

Given the heterogeneity of borderline personality disorder (BPD) patients, recent studies investigated the existence of personality subtypes in BPD patients. ${ }^{1-3}$ A person-centered approach in a theoretical framework can explain different phenotypes of BPD. Frias et al. ${ }^{1}$ identified four subtypes based on personality traits, and Smits et al. ${ }^{3}$ distinguished three subtypes based on dimensional PD features. Since temperament is a promising transdiagnostic process that underlies psychopathology, different temperament dimensions such as reactive and regulative temperament can define subtypes. Previous studies have displayed different subtypes based on temperament dimensions and their differential associations with psychopathology within various clinical samples (e.g. Müller et $\mathrm{al}^{4}$ ). Subtypes based on temperament dimensions, characterized by differential psychiatric comorbidity, may demonstrate different treatment responses. In a previous study, Sleuwaegen et al. $^{2}$ distinguished four BPD subtypes based on reactive (Behavioural Activation System reactivity; BAS and Behavioural Inhibition System reactivity; BIS) and regulative temperament (effortful control): an Emotional/Disinhibited subtype (low effortful control), characterized by the highest level of symptomatology and comorbid histrionic PD; an Inhibited subtype (low BAS), demonstrating low expression of emotions and comorbid cluster C PD; a Low Anxiety subtype (low BIS) characterized by low anxiety, high expression of emotions and comorbid antisocial PD; and a High Self-control subtype (high effortful control) showing the highest level of functioning.

The identification of BPD subtypes may be important for enhancing the effectiveness of assessment and treatment of BPD patients ${ }^{5}$; however, the clinical utility of using BPD subtypes has not yet been proven. Insight in treatment trajectories of the different BPD subtypes may support the development of more tailored treatment interventions and may guide treatment choices to optimize treatment outcome.
Several effectivity studies of dialectical behaviour therapy (DBT) inpatient treatment showed significant improvement in ratings of non-suicidal injury (NSSI), general clinical symptomatology, borderline-related symptoms and dissociation after a 3-month DBT program in BPD samples. ${ }^{6,7}$ However, a third of the patients did not benefit from treatment. ${ }^{7}$ In addition, in DBT, the dropout rate was estimated around $27 \%$, and till now few predictors of dropout have been identified (e.g. Kliem et $\mathrm{al}^{8}$ ). It is hypothesized that different BPD subtypes might show differences in treatment trajectories (e.g. Oldham ${ }^{9}$ ), explaining the mixed treatment responses of BPD patients. To our knowledge, only one study so far has evaluated differences in treatment responses between different BPD subtypes. Digre et al. ${ }^{10}$ assessed 74 BPD inpatients and identified three BPD subtypes focusing on attribution style. After 6 months of treatment, which consisted of some DBT skills training aspects, a significant improvement in depression, dissociation and less suicide attempts, but no significant change in NSSI was demonstrated. However, they did not find significant trajectory differences between the different BPD subtypes for each of these symptoms. BPD subtypes based on reactive and regulative temperament might demonstrate differences in treatment trajectories, since evidence from an earlier study found that clinical subtypes based on temperament dimensions were connected with clinical outcomes. $^{11}$

The aim of this study was threefold: (1) to replicate the prior BPD subtypes based on reactive and regulative temperament dimensions (see Sleuwaegen et $\mathrm{al}^{2}$ ) with model-based clustering (MBC), since this method only generates multiple clusters if the data provides evidence for them; (2) to investigate differences in symptomatology and coping between the BPD subtypes at baseline; and (3) to investigate the (potential differential) dropout rate and differences in symptomatology and coping of these BPD subtypes after 3 months of BPD inpatient treatment. ${ }^{12}$ Although this study was exploratory in nature, several hypotheses were 
formulated based on prior research. First, we expected to find four BPD subtypes based on temperament as in a previous study. ${ }^{2}$ Second, we expected intergroup differences in symptomatology and coping at baseline. The subtype with the highest level of BIS and BAS, and the lowest level of effortful control (Emotional/disinhibited cluster) would show the highest level of symptomatology and the worst coping compared to the other subtypes. ${ }^{2}$ Third, it was expected that the total BPD sample would show a good treatment response (main effect of time) on general clinical and borderline specific symptoms, dissociation, NSSI and coping styles (e.g. Kröger et $\mathrm{al}^{7}$ ). Since the exploration of trajectory differences among the BPD subtypes based on temperament dimensions is new, we did not formulate hypotheses concerning potential differences in symptomatology and coping after 3 months of DBT inpatient treatment. However, we hypothesized a higher dropout rate in the subtype with the lowest BIS level, consistent with past research in samples with anti-social personality characteristics. ${ }^{13}$

\section{Methods}

\section{Participants and procedure}

Our participants consisted of 145 BPD inpatients (88.3\% females) hospitalized in a treatment unit for BPD patients, based on the principles of DBT. ${ }^{12}$ Participants were included between May 2014 and April 2017. Patients were included if they fulfilled the criteria of BPD, and they were excluded if they suffered from a current psychotic disorder or a current substance use disorder. In case of current addiction, patients were referred to a specialized unit for detoxification and substance use disorder. Patients were allowed to be under pharmacological treatment. During the first week of admission, all patients were informed about the aims of the study and invited to participate. After giving written informed consent, patients were asked to fill out the questionnaires on a computer. All 145 patients met criteria for BPD diagnosis as assessed by means of the self-report Assessment of DSM-IV Personality Disorders-Borderline scale (ADP-IV; Schotte, 1994), and for 80 patients this BPD diagnosis was validated by the Structured Clinical Interview for DSM-IV Axis II Personality Disorders (SCID-II ${ }^{14}$; Dutch translation by ${ }^{15}$ ). See Table 1 for more detailed information. Assessment after 3 months ( 13 to 16 weeks) was carried out as an element of routine outcome monitoring which is integrated into the regular treatment routine to evaluate treatment response. After 3 months of DBT treatment, 75 inpatients provided data concerning clinical symptomatology and coping. Table 1 displays the sample characteristics for the sample at T1 and the sample at T2. The study was developed in accordance with the Declaration of Helsinki and approved by the local research and participating hospitals ethics committees. Participants did not receive any remuneration.

Table 1: Sample characteristics for the total sample of BPD patients at baseline and for the subsample at 3 months assessment

\begin{tabular}{|c|c|c|}
\hline & $\begin{array}{c}\text { Total sample } \\
\text { at T1 }\end{array}$ & $\begin{array}{l}\text { Sample } \\
\text { at T2 }\end{array}$ \\
\hline & $n=145$ & $n=75$ \\
\hline Gender-Female (\%) & 88.3 & 91.9 \\
\hline Age $(M, S D)$ & $29.72(9.32)$ & $28.75(8.83)$ \\
\hline \multicolumn{3}{|l|}{ Education (\%) } \\
\hline Lower secondary & 19.7 & 18.9 \\
\hline Higher secondary & 57.8 & 55.4 \\
\hline High school & 17.6 & 18.9 \\
\hline University & 4.7 & 6.8 \\
\hline \multicolumn{3}{|l|}{ Marital status (\%) } \\
\hline Single & 71.4 & 71.6 \\
\hline Living together/married & 19.7 & 18.9 \\
\hline Divorced & 8.8 & 9.5 \\
\hline Medication (\%) & 84.8 & 85.1 \\
\hline Antidepressants & 71.7 & 70.3 \\
\hline Antipsychotics & 48.3 & 44.6 \\
\hline Anxiolytics & 6.2 & 6.8 \\
\hline Mood stabilizers & 11.7 & 10.8 \\
\hline
\end{tabular}

(Note. Antipsychotics provided as mood regulator) 


\section{Measures}

The diagnosis of BPD was assessed by means of the Assessment of DSM-IV Personality Disorders (ADP-IV ${ }^{16}$ ), a 94-item Dutch self-report questionnaire used to assess the presence and severity of the personality disorders as defined in the DSM-IV-TR ${ }^{17}$ and the Structured Clinical Interview for DSM-IV Axis II Personality Disorders (SCID-II ${ }^{14}$; Dutch translation by Weertman et $\mathrm{al}^{15}$ ). Schotte et al. ${ }^{18}$ indicated that the ADP-IV-BPD scale showed acceptable concordance with the SCID-II-BPD section $($ kappa $=0.54)$.

To replicate the BPD subtypes based on reactive/regulative temperament, we used the Behavioural Inhibition System/Behavioural Activation System Scales (BIS/BAS ${ }^{19}$; Dutch translation $^{20}$ ) and the Effortful Control Scale (ECS) of the Adult Temperament QuestionnaireShort Form. ${ }^{21}$ The BISBAS consist of 24-items, rated on a 4-point Likert scale, of which seven items assess BIS reactivity ( $\alpha=.72$ in the present study), reflecting sensitivity to punishment, and 13 items assess BAS reactivity ( $\alpha=.86)$, reflecting sensitivity to potentially rewarding outcome. Regulative temperament was assessed by means of the ECS with 19 items rated on a seven-point Likert scale. The internal consistency of the ECS was $\alpha=.77$ in the present sample.

To validate the BPD subtypes and to investigate treatment response, several self-report measures were administered assessing clinical/BPD symptomatology and coping:

General clinical symptomatology was assessed by means of the Brief Symptom Inventory (BSI ${ }^{31}$; translated into Dutch by De Beurs ${ }^{22}$ ) consisting of 53 items, rated on a 4-point Likert scale. In the present study, the alpha coefficient for the total BSI-score was.95.

Borderline specific symptoms were measured by means of the Borderline Symptom List-23 $\left(\right.$ BSL- $\left.23^{23}\right)$, in which patients rated 23 items on a 5-point Likert scale. Cronbach's alpha of the total BSL-score in the present study was .95 .
Dissociation was assessed by means of the Dissociation Questionnaire (DIS- $Q^{24}$ ), consisting of 63 -items to be rated on a 5-Likert scale. In this study, the internal consistency coefficient of the total DIS-Q-score was excellent $(\alpha=.96)$.

Non-suicidal self-injury was assessed by means of the Self-Injury Questionnaire-Treatment Related. ${ }^{25}$ Participants had to answer in a yes/no question format whether they had ever intentionally engaged in self-injury without the intention to die (i.e. scratching, cutting, hitting, burning, picking or head banging) (life-time NSSI) and whether they were still engaging in NSSI at the current moment (current NSSI). ${ }^{25}$

Coping strategies were assessed by means of the Utrecht Coping List $\left(\mathrm{UCL}^{26}\right)$, consisting of 47 items, to be rated on a 5-point Likert scale. Two coping styles can be distinguished: an active coping style (based on the subscales 'active problem solving' and 'self-soothing thoughts') and a passive coping style (based on the subscales 'avoidance', 'depressive reactions' and 'expression of emotions $\left.{ }^{26}\right)$. In this study, the alpha coefficient for active coping style was .84 and for passive coping style 69 .

\section{Statistical analysis}

Statistical analyses were performed with SPSS version 24 and $\mathrm{R}$ version 3.4.3. There were no missing data given that the assessment on the computer did not allow for missing values.

To identify different BPD subtypes based on reactive and regulative temperament dimensions, we performed a model-based cluster analysis ${ }^{27}$ on the standardized BIS/BAS and EC scale scores (i.e. $z$-scores) using the mclust package version 5.4 (Scrucca et al., 2016). Compared to standard clustering techniques such as hierarchical clustering and $\mathrm{k}$-means, $\mathrm{MBC}$ allows for more flexible cluster distributions in terms of their volume, shape and orientation. As such, MBC, in general, yields cluster solutions of a higher quality. In particular, MBC fits a mixture of Gaussian distributions to the data and searches for the best 
model across a predefined set of models that differ in the volume, shape and orientation of their cluster distributions (see Scrucca et al., 2016). In our analysis, 10 different models were tested with the number of clusters ranging from one to six (60 models in total). To overcome convergence problems when estimating the parameters of some of these models, we applied a Bayesian regularization as recommended in Fraley and Raftery. ${ }^{28}$ To choose the optimal model, we mainly relied on the Bayesian information criterion (BIC) but also took interpretability of the solution into account; BIC balances model fit with model complexity (i.e. number of clusters and complexity of the cluster distributions). We additionally compared the best $\mathrm{MBC}$ cluster solution with solutions obtained from the hierarchical cluster analysis and $\mathrm{k}$-means clustering procedure.

To compare the different BPD-subtypes with respect to general clinical symptomatology, BPD specific symptomatology, dissociation and coping styles, we performed a MANOVA with BPD subtypes as independent variable and clinical, BPD symptomatology, dissociation and coping as dependent variables. The association between categorical variables (presence/absence of NSSI) and BPD subtype at baseline was analysed using the Chi-square test statistic.

To investigate the association between the presence/absence of dropout and BPD subtypes, we used the Chi-square test statistic. In addition, to evaluate differences in the level of general clinical symptomatology, BPD specific symptomatology, dissociation and two coping styles at baseline and after 3 month of treatment in function of BPD subtype, we performed five repeated measures ANOVAs with time (baseline and 3 months) as within-subject factor, BPD subtype as between-subject factor, and general clinical symptomatology, BPD symptomatology, dissociation and two coping styles as dependent variables.

All significant group differences were evaluated using the post hoc Bonferroni test. $P<.05$ was considered statistically significant.
Partial eta-squared value was reported as a measure of effect size. ${ }^{29}$

To calculate the association between BPD subtype and NSSI throughout time, we used the Chi-Square test statistic. NSSI was coded as follows: 0 = no NSSI baseline - no NSSI $3 \mathrm{~m}$; $1=$ NSSI baseline - no NSSI $3 \mathrm{~m} ; 2$ = NSSI baseline - NSSI $3 \mathrm{~m}$.

\section{Results}

BPD subtype clusters

BIS/BAS and EC scores were analysed by means of a model-based cluster analysis to identify BPD subtypes. The three best fitting models using BIC were as follows: a VVI (diagonal, varying volume and shape) model with two components, BIC $=-1202.079 ;$ a VVV (ellipsoidal, varying volume, shape and orientation) model with two components, $\mathrm{BIC}=-1205.934$ and a VVI model with three components BIC $=-1216.226$. The other models all had a worse BIC value (BIC $<-1231.189)$. The two best fitting models (VVI and VVV with two clusters) gave very similar results and both clustered the patients into two groups that only differ in BIS level. As these clusters are not very heterogeneous in terms of temperamental dimensions, which was the goal of our study, we elaborated further on the third best fitting model, the three-cluster model (VVI-3). This model resulted in clusters with a more distinguished profile regarding the three temperamental dimensions (BIS/BAS/EC). As, to our opinion, this solution is readily interpretable and clinically more relevant than the best fitting solution, we decided to prefer the VVI model with three clusters over the best fitting VVI2 model. Note that the difference in BIC between both models is rather small. Moreover, the additional analyses with hierarchical and $\mathrm{k}$-means clustering also favoured a three-cluster solution.

The three BPD clusters showed substantively different means on the BIS/BAS and EC scale (see Figure 1). The first cluster was identified as 


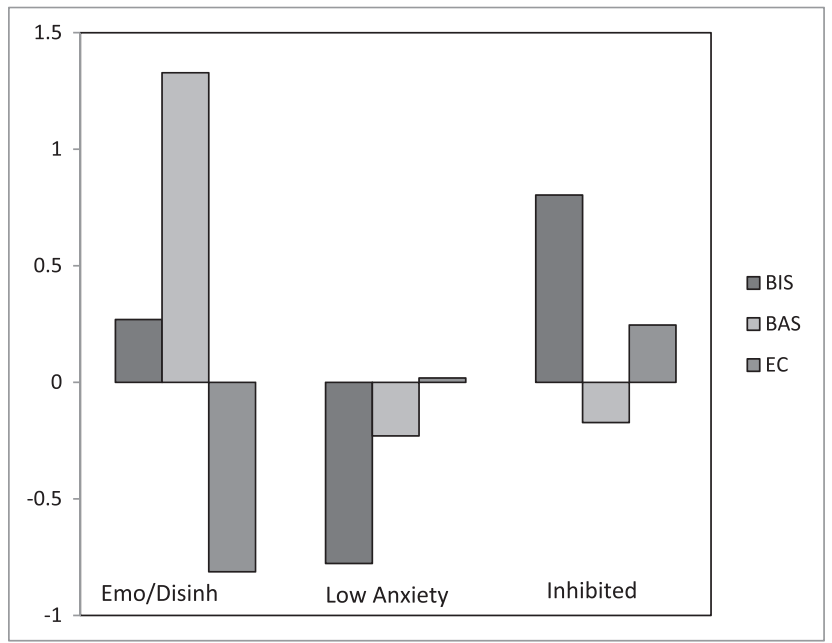

Note: BIS $=$ Behavioural Inhibition System; BAS $=$ Behavioural Activation System; EC $=$ Effortful Control BPD Subtypes $:$ Emo/Dis $=$ Emotional/Disinhibited, Low Anx $=$ Low Anxiety, Inh $=$ Inhibited

Figure 1: BPD subtypes based on reactive and regulative temperament dimensions in $145 \mathrm{BPD}$ patientsNote: BIS = behavioural inhibition system; $\mathrm{BAS}=$ behavioural activation system; $\mathrm{EC}=$ effortful controlBPD subtypes: $\mathrm{Emo} / \mathrm{Dis}=$ emotional/ disinhibited, Low Anx = low anxiety, Inh = inhibited

the 'Emotional/Disinhibited' subtype $(n=22$, $15 \%)$, characterized by moderately high scores on BIS, high scores on BAS and low scores on EC. The second cluster was named the 'Low Anxiety subtype' $(n=59,41 \%)$, characterized by very low BIS, average low BAS and neutral EC scores. The third and last cluster was called the 'Inhibited subtype' ( $n=64,44 \%)$, characterized by a high score on BIS, a low score on BAS and moderate score on EC. Note that the two clusters from the best fitting model (VVI2) are closely related to the 'Low Anxiety subtype' and the 'Inhibited subtype', with the former subtype having a low BIS and the latter one a high BIS score (and subtypes not differing on BAS and EC scores). As such, the adopted three-cluster solution identified a new subtype-the 'Emotional/Disinhibited' subtype-with a clearly different reactive/regulative temperament, which justifies adopting the threecluster solution instead of the slightly better fitting two-cluster solution. The patients in the three clusters did significantly differ with regard to gen$\operatorname{der}\left[\chi^{2}(2)=10.22, p=.006\right]$ with the Low Anxiety subtype containing relatively more males
(22\% male and $78 \%$ female) than the 'Emotional/Disinhibited' (4.5\% males) and the Inhibited subtype ( $4.7 \%$ males). There were no significant difference between the three subtypes for age $[F(3,142)=.60, p=.55]$, education $\left[\chi^{2}(6)=2.96, p=.81\right]$, marital status $\left[\chi^{2}(4)=.81, p=.94\right]$ and overall medication use [yes/no: $\chi^{2}(2)=1.16, p=.56$ ]

Differences in symptomatology and coping of the three BPD subtypes at baseline

Table 2 lists the means and standard deviations of the different measures for each of the three BPD subtypes. The MANOVA showed only significant differences among the three BPD subtypes with respect to dissociation (total DIS-Q). The Emotional/Disinhibited subtype scored significant higher on the DIS-Q compared to the Inhibited subtype $(p=.002)$, with a moderate effect size (partial $\eta^{2}=.089$ ).

There was no significant difference between the three BPD subtypes for presence of lifetime NSSI [Emotional/Disinhibited subtype: 81.8\%, Low 
Table 2: Means (standard deviations) of the self-report scales at baseline for the three BPD subtypes

\begin{tabular}{lcccccc}
\hline & $\begin{array}{c}\text { Emotional/disinhibited } \\
\text { subtype }(n=22)\end{array}$ & $\begin{array}{c}\text { Low anxiety } \\
\text { subtypes }(n=59)\end{array}$ & $\begin{array}{c}\text { Inhibited subtype } \\
(n=64)\end{array}$ & & & \\
& $\mathrm{M}(\mathrm{SD})$ & $\mathrm{M}(\mathrm{SD})$ & $\mathrm{M}(\mathrm{SD})$ & $\mathrm{F}$ & Partial $\eta^{2}$ & Post-hoc comparisons \\
\hline BSI & $123.59(26.77)$ & $108.78(38.71)$ & $122.02(32.3)$ & 2.80 & .04 & \\
BSL & $50.55(23.49)$ & $48.78(21.92)$ & $55.02(19.09)$ & 1.39 & .02 & \\
DIS-Q & $199.32(25.87)$ & $163.97(42.70)$ & $165.17(42.32)$ & $6.89^{*}$ & .09 & $1<2,3$ \\
UCL-ACT & $23.23(7.02)$ & $22.34(6.19)$ & $22.06(5.01)$ & .33 & .05 & \\
UCL-PASS & $49.23(5.73)$ & $46.58(6.74)$ & $46.56(6.28)$ & 1.61 & .02 & \\
& & & & & & \\
\hline
\end{tabular}

Note. BSI = Brief Symptom Inventory-total score; BSL $=$ Borderline Symptom List-total score; DIS-Q = Dissociation Questionnaire; UCL-ACT = Utrecht Coping List—Active coping style score; UCL-PASS = Utrecht Coping List—Passive coping style score.

$* p<0.05$.

Anxiety: $84.7 \%$, Inhibited: $79.7 \% ; \chi^{2}(2)=.53$, $p=.765$ ], nor for presence of current NSSI [resp. $\left.51.6 \%, 61.0 \%, 36.4 \% ; \chi^{2}(2)=4.02, p=.134\right]$.

Finally, the three BPD subtypes showed no significant difference on the UCL active and passive coping styles (resp. $p=.72$ and $p=.20$ ).

Difference in dropout and treatment response after 3 months in the three BPD-subtypes

After 3 months of DBT inpatient treatment, $34.5 \%(n=50)$ of all patients dropped out. There was no significant difference between the three
BPD subtypes and the presence/absence of dropout [Emotional/Disinhibited subtype: 27.3\% $(n=6)$, Low Anxiety subtype: $39 \%(n=23)$, and Inhibited subtype: $32.8 \%(n=21)$; $\left.\chi^{2}(2)=1.11, p=.573\right]$.

Almost $80 \%$ of the 95 patients who were still in treatment completed the second measurement $(n=75)$. Table 3 displays the means and standard deviations of the different self-report measures at baseline and after 3 months of DBT treatment for each of the three BPD subtypes, and Table 4 lists the results of each of the repeated measures ANOVAs.

Table 3: Means (standard deviations) of the self-report scales at baseline (T1) and after 3 months (T2) for the three BPD subtypes

\begin{tabular}{|c|c|c|c|c|c|c|}
\hline & \multicolumn{2}{|c|}{ Emotional/disinhibited subtype $(n=11)$} & \multicolumn{2}{|c|}{ Low anxiety subtype $(n=27)$} & \multicolumn{2}{|c|}{ Inhibited subtype $(n=37)$} \\
\hline & $\mathrm{T} 1$ & $\mathrm{~T} 2$ & $\mathrm{~T} 1$ & $\mathrm{~T} 2$ & $\mathrm{~T} 1$ & $\mathrm{~T} 2$ \\
\hline & $\mathrm{M}(\mathrm{SD})$ & $\mathrm{M}(\mathrm{SD})$ & $\mathrm{M}(\mathrm{SD})$ & $\mathrm{M}(\mathrm{SD})$ & $\mathrm{M}(\mathrm{SD})$ & $\mathrm{M}(\mathrm{SD})$ \\
\hline BSI & $134.91(17.77)$ & $93.73(18.69)$ & $99.37(33.12)$ & $93.20(42.70)$ & $125.05(30.90)$ & $98.81(41.64)$ \\
\hline BSL & 63.18 (17.71) & $41.33(14.54)$ & $47.35(20.28)$ & $42.96(23.65)$ & 55.35 (19.03) & $45.97(24.14)$ \\
\hline DIS-Q & $207.82(29.02)$ & $164.91(36.70)$ & $154.30(40.71)$ & $143.59(38.48)$ & $162.97(44.70)$ & $146.00(48.61)$ \\
\hline UCL-ACT & $24.64(7.23)$ & $29.64(7.70)$ & $21.26(5.75)$ & $23.56(5.50)$ & $22.81(5.46)$ & $26.81(5.85)$ \\
\hline UCL-PASS & $50.18(5.31)$ & $43.91(6.09)$ & $45.26(6.02)$ & $43.19(6.94)$ & 45.27 (6.68) & $42.08(7.02)$ \\
\hline
\end{tabular}

Note. BSI = Brief Symptom Inventory-total score; BSL = Borderline Symptom List-total score; DIS-Q = Dissociation Questionnaire; UCL-ACT = Utrecht Coping List—Active coping style score; UCL-PASS = Utrecht Coping List—Passive coping style score. 
Table 4: Results for the repeated measurement ANOVA with time as within-subject factor, BPD subtype as between-subject factor, and clinical, BPD symptomatology and coping as dependent variables

\begin{tabular}{|c|c|c|c|c|c|c|c|c|c|}
\hline & \multicolumn{2}{|c|}{ Main effects time } & \multirow[b]{2}{*}{ Partial $\eta^{2}$} & \multicolumn{2}{|c|}{ Main effects subtype } & \multirow[b]{2}{*}{ Partial $\eta^{2}$} & \multicolumn{3}{|c|}{ Interaction effect time * subtype } \\
\hline & $F(1,144)$ & $p$ & & $F(2,143)$ & $p$ & & $F(2,143)$ & $p$ & Partial $\eta^{2}$ \\
\hline BSI & 35.78 & $<.001$ & .335 & 2.91 & .130 & .056 & 4.96 & .010 & .123 \\
\hline BSL & 21.19 & $<.001$ & .243 & 1.29 & .280 & .038 & 3.96 & .024 & .107 \\
\hline DIS-Q & 27.07 & $<.001$ & .273 & 3.79 & .027 & .088 & 3.49 & .036 & .088 \\
\hline UCL-ACT & 24.18 & $<.001$ & .251 & 3.65 & .031 & .092 & 1.07 & .348 & .029 \\
\hline UCL-PASS & 19.12 & $<.001$ & .210 & 1.51 & .227 & .040 & 1.53 & .222 & .041 \\
\hline
\end{tabular}

Note. BSI = Brief Symptom Inventory—total score; BSL = Borderline Symptom List—total score; DIS-Q = Dissociation Questionnaire; UCL-ACT = Utrecht Coping List—Active coping style score; UCL-PASS = Utrecht Coping List—Passive coping style score.

We found significant main effects of time for all the self-report measures. The results clearly showed a decrease in general clinical symptomatology (BSI), borderline specific symptomatology (BSL), dissociation (DIS-Q) and passive coping style (UCL-PASS); and a significant increase in active coping style (UCL-ACT) from baseline till 3 months after DBT treatment with all large effect sizes (ranging from partial $\eta^{2}=.210$ to.335).

Additionally, a significant interaction effect of time by BPD subtype was observed for BSI

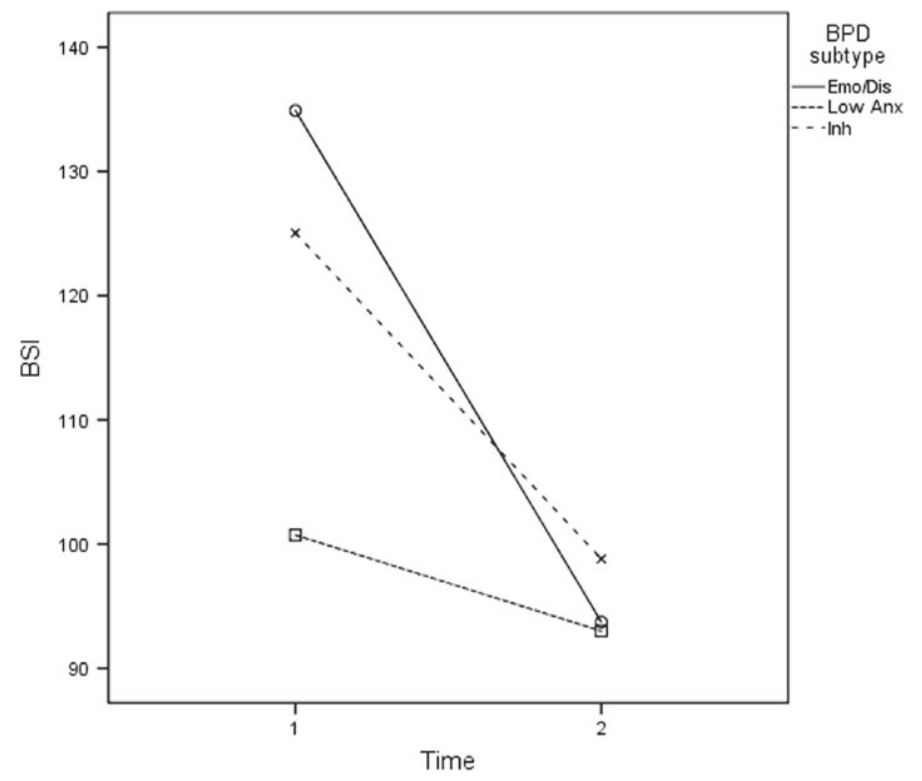

Note: $\mathrm{BSI}=$ Brief Symptom Inventory, T1 = baseline; T2 $:$ after 3 months DBT inpatient treatment BPD Subtypes $:$ Emo/Dis $=$ Emotional/Disinhibited, Low Anx $=$ Low Anxiety, Inh $=$ Inhibited

Figure 2: Reduction of general clinical symptoms after 3 months DBT treatment for the three BPD subtypesNote: BSI $=$ brief symptom inventory, T1 = baseline; T2: after 3 months DBT inpatient treatmentBPD subtypes: Emo/Dis = emotional/ disinhibited, Low Anx = low anxiety, Inh = inhibited 
$(p=.010)$ and BSL $(p=.024)$ with large effect sizes (resp. partial $\eta^{2}=.123$ and .107), and DIS$Q(p=.036)$ with a moderate effect size (partial $\eta^{2}=.088$ ), see Table 4 . Figures 2,3 and 4 respectively illustrate the progress of general clinical symptoms, borderline specific symptoms and dissociation in the three BPD subtypes after 3 months of DBT treatment. It should be noted that patients of the 'Emotional/Disinhibited' subtype shows a larger progress on these measures, indicating that it is justified to consider this patient cluster as a separate subtype.

Since the interaction effect of time by subtype was significant for BSI (clinical symptoms), BSL (borderline symptoms) and DIS-Q (dissociation), we investigated the time effect (baseline3 months) on the different symptom measures within each subtype using paired samples t-tests. For the Emotional/Disinhibited subtype, a significant decrease was found for BSI $[t(10)=5.97$, $p<.001]$, BSL $[t(10)=4.35, p=.002]$ and DIS$Q[t(10)=2.75, p=.021]$. For the Low Anxiety subtype, no significant difference was found in symptom measurements between baseline and 3 months (BSI $[t(26)=1.46, p=.156]$, BSL $[t(26)=1.15, p=.260]$, DIS-Q $[t(26)=1.77$, $p=.088])$. For the Inhibited subtype, a significant decrease was found for BSI $[t(36)=4.36$, $p<.001]$, BSL $[t(36)=2.38, p=.023]$ and DIS-Q $[t(36)=3.41, p=.002]$.

In addition, no significant interaction effects of 'time by subtype' were demonstrated for UCL active and passive coping style (resp. $p=.35$ and $p=.22)$.

Finally, of all BPD patients who engaged in NSSI at baseline, $60.0 \%$ of the Emotional/Disinhibited subtype, $60.9 \%$ of the Low Anxiety and $62.5 \%$ of the Inhibited reported the absence of current NSSI at 3 months follow-up. Analysing the trajectory on current NSSI from baseline to 3 months after treatment for the three subtypes revealed no difference in NSSI trajectory between the BPD subtypes $\left[\chi^{2}(4)=4.97, p=.290\right]$, indicating that the different subtypes showed the same improvement for NSSI.

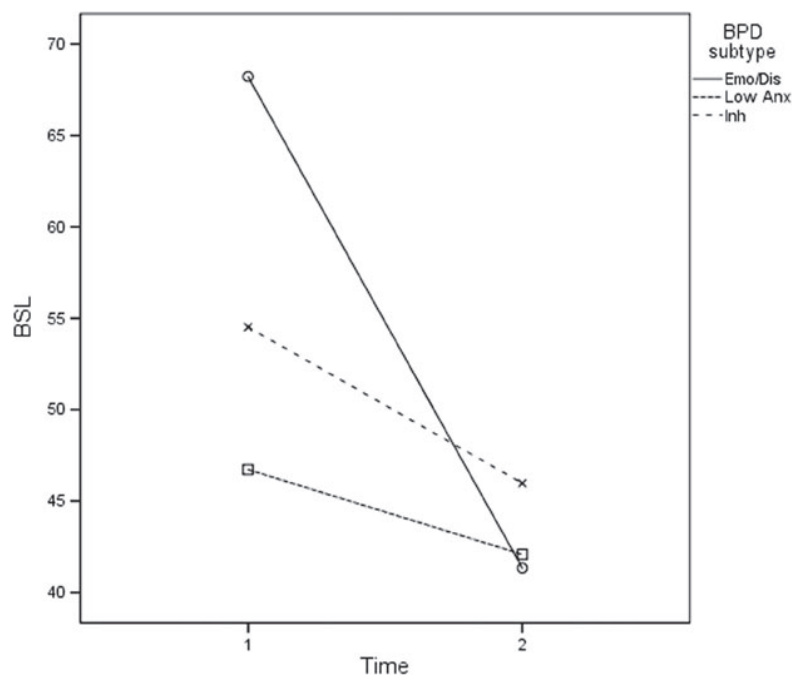

Note: $\mathrm{BSL}=$ Borderline Symptom List, $\mathrm{T} 1=$ baseline; $\mathrm{T} 2:$ after 3 months DBT inpatient treatment BPD Subtypes : Emo/Dis = Emotional/Disinhibited, Low Anx = Low Anxiety, Inh = Inhibited

Figure 3: Reduction of borderline specific symptoms after 3 months DBT treatment for the three BPD subtypesNote: $\mathrm{BSL}=$ borderline symptom list, T1 = baseline; T2: after 3 months DBT inpatient treatmentBPD subtypes: Emo/Dis = emotional/disinhibited, Low Anx = low anxiety, Inh = inhibited 


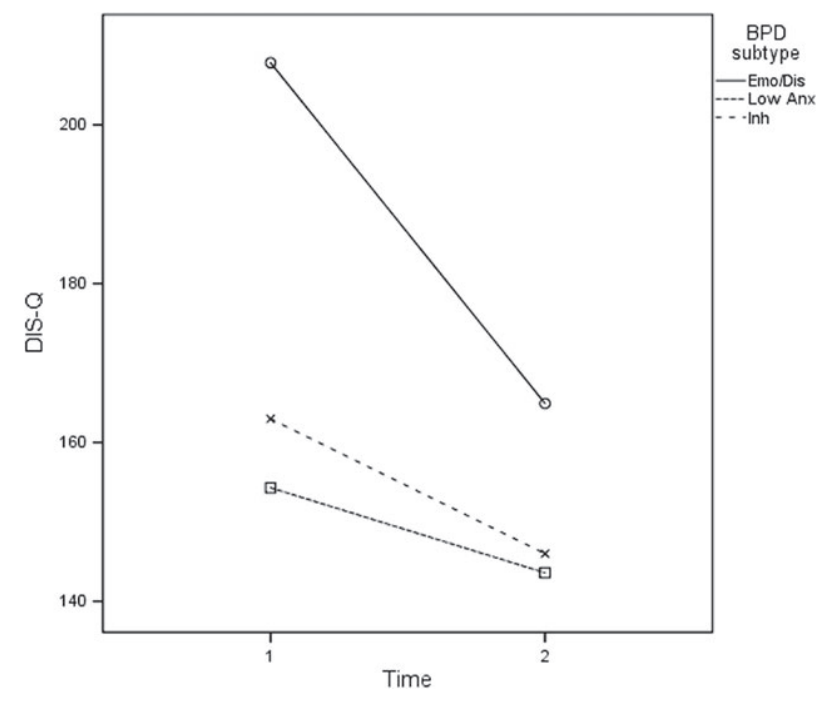

Note: DIS-Q $=$ Dissociation Questionnaire, $\mathrm{T} 1=$ baseline; $\mathrm{T} 2:$ after 3 months DBT inpatient treatment BPD Subtypes : Emo/Dis $=$ Emotional/Disinhibited, Low Anx $=$ Low Anxiety, Inh $=$ Inhibited

Figure 4: Reduction of dissociation symptoms after 3 months DBT treatment for the three BPD subtypesNote: DIS-Q = dissociation questionnaire, $\mathrm{T} 1$ = baseline; T2: after 3 months DBT inpatient treatmentBPD subtypes: Emo/Dis $=$ emotional/ disinhibited, Low Anx = low anxiety, Inh = inhibited

\section{Discussion}

The aim of the present study was to confirm the existence of BPD subtypes based on temperament, to validate them in terms of symptomatology and coping and to investigate their differences in treatment response after 3 months of inpatient DBT. To replicate previously identified BPD subtypes, we performed a model-based cluster analysis on reactive and regulative temperament dimensions in a sample of $145 \mathrm{BPD}$ patients who were treated at a DBT unit. We identified three subtypes, the 'Emotional/Disinhibited', 'Low Anxiety' and 'Inhibited' subtypes, demonstrating the same characteristics as the three dominant subtypes found in a previous study using a hierarchical and $\mathrm{k}$-means clustering procedure (see Sleuwaegen et $\left.\mathrm{al}^{2}\right)$. The fourth and smallest cluster found in the previous study, namely the 'High Self-control subtype' (characterized by high scores on EC and moderately high scores on BIS and BAS) was not found in this sample. In this sample, the
Inhibited subtype is also characterized by low levels of BAS reactivity (low reward sensitive) and moderate effortful control; however, a higher level of BIS was demonstrated. The Low Anxiety subtype is characterized by very low levels of BIS reactivity (low punishment sensitive) and low levels of BAS and a mean level of effortful control. The Emotional/Disinhibited subtype is characterized by a high level of BAS, moderate BIS and low effortful control. Patients of the Emotional/Disinhibited subtype reported a higher level of dissociation compared to the other two BPD subtypes. There was no difference in lifetime and current NSSI between the different BPD-subtypes.

The third, and most clinically relevant, aim of this study was to investigate differences in dropout and treatment response of these three BPD subtypes after 3 months of inpatient DBT treatment. Dropout rate in the total sample was $34.5 \%$; however, there was no significant difference in amount of dropout between the three 
BPD subtypes. Trajectory measurement on data of 75 patients who filled out the questionnaires at baseline and after 3 months DBT inpatient treatment demonstrated a significant reduction in general clinical symptoms, borderline specific and dissociative symptoms with large effect sizes in all BPD subtypes. Moreover, there was a significant interaction effect (time by BPD subtype) on general clinical and borderline specific symptoms and dissociation, explaining a different evolution between the three subtypes on the level of symptomatology. The results demonstrated that, in contrast to the Emotional/Disinhibited subtype and Inhibited subtype, the Low Anxiety subtype did not improve significantly in general clinical and borderline specific symptoms and dissociation. These aforementioned results are in line with a previous study of Kröger et al., ${ }^{7}$ who also reported a reduction in clinical symptoms in almost all patients after a 3-month DBT program, but for borderline-specific symptoms only for half of them.

In the present study, almost $80 \%$ of the patients reported to engage in NSSI at baseline, and 60\% of these patients did not engage in NSSI anymore after 3 months of DBT inpatient treatment. However, the reduction of NSSI over time was not significant, and there was no difference in NSSI trajectory between the three BPD subtypes, which is in line with the findings of Digre et al. ${ }^{10}$ Finally, concerning coping style, all BPD patients showed an increase in active coping and a decrease in passive coping after 3 months of treatment. However, there was no evidence for significant differences in coping progress between the three BPD subtypes.

However, our study is not without limitations which need to be taken into account in future studies. The first limitation refers to the short follow-up period of 3 months of treatment and high drop-out rate. Therefore, future studies should try to include more patients followed during a longer time period, to assess the treatment trajectories of different BPD subtypes. A second limitation is the fact that the data were collected in a specialized DBT unit, overrepresented by female and medicated patients, which makes the sample not representative for the general BPD population. As a third limitation, all baseline/outcome assessments were solely based on self-report measures, with the known limitations. ${ }^{30}$ In addition, since we have only patients with BPD in the sample, the associations between the temperamental dimensions may not be representative for other samples.

In conclusion, the present study confirms the existence of BPD subtypes based on reactive and regulative temperament. Furthermore, in addition to the finding that all subtypes show significant improvement in level of symptomatology and coping after 3 months of DBT inpatient treatment, this study provides evidence for trajectory differences on general clinical and borderline specific symptoms and dissociation between the three subtypes. The Low Anxiety subtype does not demonstrate a significant reduction in vsymptomatology compared to the two other subtypes. This finding is an incentive for future research on the trajectory of BPD subtypes based on temperament, in larger samples and with longer follow-up periods. The conclusions may guide treatment choices and/or the development of subtype-tailored treatment in the future, which would be of clinical and social benefit.

\section{Acknowledgements}

This study was partly supported by the Research Foundation Flanders (FWO), thanks to a Special PhD fellowship (1901317N).

\section{References}

1. Frias A, Navarro S, Palma C, Farraiols N, Alagia F, Salvador A et al. Defining subtypes of borderline personality disorder based on underlying dimensional personality traits. Personal Ment Health 2017; 27: $25-30$. 
2. Sleuwaegen E, Claes L, Luyckx K, Berens A, Vogels C, Sabbe B. Subtypes in borderline patients based on reactive and regulative temperament. Personal Individ Differ 2017; 108: 14-9.

3. Smits ML, Feenstra DJ, Bales DL, de Vos J, Lucas Z, Verheul R et al. Subtypes of borderline personality disorder patients: a cluster-analytic approach. Borderline Personality Disorder and Emotion Dysregulation 2017; 4(16): 1-15.

4. Müller A, Claes L, Wilderjans $\mathrm{T}$, de Zwaan $\mathrm{M}$. Temperament subtypes in treatment seeking obese individuals: a latent profile analysis. Eur Eat Disord Rev 2014; 22: 260-6.

5. Kopala-Sibley DC, Zuroff DC, Russell JJ, Moskowitz DS, Paris J. Understanding heterogeneity in borderline personality disorder: differences in affective reactivity explained by the traits of dependency and self-criticism. J Abnorm Psychol 2012; 121: 680-91.

6. Bohus M, Haaf B, Simms T, Limberger MF, Schmahl C, Unckel C et al. Effectiveness of inpatient dialectical behavioral therapy for borderline personality disorder: a controlled trial. Behav Res Ther 2004; 42: 487-99.

7. Kröger C, Harbeck S, Ambrust W, Kliem S. Effectiveness, response and dropout of dialectical behavior therapy for borderline personality disorder in an inpatient setting. Behav Res Ther 2013; 51: 411-16.

8. Kliem S, Kroger C, Kosfelder J. Dialectical behavior therapy for borderline personality disorder: a meta-analysis using mixed-effects modeling. J Consult Clin Psychol 2010; 78: 936-51.

9. Oldham JM. Borderline personality disorder and suicidality. Am J Psychiatry 2006; 163: 20-26.

10. Digre EI, Reece J, Johnson AL, Thomas RA. Treatment response in subtypes of borderline personality disorder. Personal Ment Health 2009; 3: 56-67.

11. Karalunas SL, Fair D, Musser ED, Aykes K, Iyer SP, Nigg JT. Subtyping attention-deficit/hyperactivity disorder using temperament dimensions: toward biologically based nosologic criteria. JAMA Psychiat 2014; 71: 1015-24.

12. Linehan, M. Cognitive-Behavioral Treatment of Borderline Personality Disorder. New York, NY: The Guilford Press, 1993.

13. Daughters SB, Stipelman BA, Sargeant MN, Schuster R, Bornovalova MA, Lejuez $\mathrm{C}$ et al. The interactive effects of antisocial personality disorder and court-mandated status on substance abuse treatment dropout. J Subst Abuse Treat 2008; 34: 157-64.

14. First MB, Gibbon M, Spitzer RL, Williams JBW, Benjamin LS. Structured Clinical Interview for DSM-IV Axis II Disorders. Washington, DC: American Psychiatric Press, 1997.
15. Weertman A, Arntz A, Kerkhofs MLM. Gestructureerd diagnostisch interview voor DSM-IV persoonlijkheidsstoornissen (SCID-II) [Structured Clinical Interview for DSM-IV Personality Disorders (SCID-II)]. Lisse: Swets Test, 2000.

16. Schotte CKW, \& De Doncker D. ADP-IV vragenlijst. Antwerpen: Universitair Ziekenhuis Antwerpen, 1994.

17. American Psychiatric Association. Diagnostic and Statistical Manual of Mental Disorders, 4th ed., text revision Edition. Washington, DC: APA, 2000.

18. Schotte CKW, De Doncker D, Dmitruk D, Van Mulders I, D'Haenen H, Cosyns P. The ADP-IV questionnaire: differential validity and concordance with semistructured interview. J Pers Disord 2004; 18: 405-19.

19. Carver CS, \& White TL. Behavioral inhibition, behavioral activation, and affective responses to impending reward and punishment: the BIS/BAS scales. J Pers Soc Psychol 1994; 67: 319-33.

20. Franken IHA, Muris P, Rassin E. Psychometric properties of the Dutch BIS/BAS scales. J Psychopathol Behav Assess 2005; $27: 25-30$.

21. Evans DE, \& Rothbart MK. Developing a model for adult temperament. J Res Pers 2007; 41: 868-88.

22. De Beurs E. De Brief Symptom Inventory; Handleiding [The Brief Symptom Inventory; Manual]. Leiden: Pits Publishers, 2005.

23. Bohus M, Kleindienst N, Limberger MF, Stieglitz RD, Domsalla M, Chapman A et al. The short version of the borderline symptom list (BSL-23): development and initial data on psychometric properties. Psychopathology 2009; 42: 32-39.

24. Vanderlinden J, Van Dyck R, Vandereycken W, Vertommen H, Verkes R, J. The dissociation questionnaire (DIS-Q): development and characteristics of a new self-report questionnaire. Clin Psychol Psychother 1993; 1: 21-27.

25. Claes L, \& Vandereycken W. The self-injury questionnaire-treatment related (SIQ-TR): construction, reliability, and validity in a sample of female eating disorder patients. In: Goldfarb PM (ed). Psychological Tests and Testing Research Trends, pp. 111-39. New York, NY: Nova Science Publishers, 2007.

26. Schreurs PJG, van de Willige G, Brosschot JF, Tellegen B, Graus GMH. De Utrechtse Coping Lijst: UCL [The Utrecht Coping List: UCL]. Lisse: Swets \& Zeitlinger, 1993.

27. Fraley C, \& Raftery AE. How many clusters? Which clustering method? Answer via model-based cluster analysis. Comput J 1998; 41: 578-88.

28. Fraley C, \& Raftery AE. Enhanced software for model-based clustering, discriminant analysis, and density estimation: MCLUST. J Classif 2003; 20: 263-86. 
29. Cohen J. Statistical Power Analysis for the Behavioural Sciences, 2nd Edition. Hillsdale, NJ: L. Erlbaum associates, 1988.

30. McDonald JD. Measuring personality constructs: the advantages and disadvantages of self-reports, informant reports and behavioural assessments. Enquire 2008; 1: 75-94.

31. Derogatis, L \& Melisaratos, N. The brief Symptom Inventory: An introductory report. The Journal of Psychological Medicine, 1983, 13: 595-605.

\section{Supporting information}

Additional supporting information may be found online in the Supporting Information section at the end of the article.

Address correspondence to: Ellen Sleuwaegen (dra.), University Department of Psychiatry, Campus Psychiatric Hospital Duffel, Duffel, Belgium. Email: ellen.sleuwaegen@uantwerpen.be 